\title{
Fast tomographic methods for the tokamak ISTTOK
}

\author{
P. J. Carvalho*, H. Thomsen ${ }^{\dagger}$, S. Gori**, U. v. Toussaint**, A. Weller ${ }^{\dagger}$, R. \\ Coelho*, A. Neto*, T. Pereira*, C. Silva* and H. Fernandes* \\ *Associação Euratom/IST Centro de Fusão Nuclear, Instituto Superior Técnico 1049-001 Lisboa, \\ Portugal \\ ${ }^{\dagger}$ Max-Planck-Institut für Plasma Physik, EURATOM Ass., 17491 Greifswald, Germany \\ ${ }^{* *}$ Max-Planck-Institut für Plasma Physik, EURATOM Ass., 85748 Garching, Germany
}

\begin{abstract}
The achievement of long duration, alternating current discharges on the tokamak ISTTOK requires a real-time plasma position control system. The plasma position determination based on magnetic probes system has been found to be inadequate during the current inversion due to the reduced plasma current. A tomography diagnostic has been therefore installed to supply the required feedback to the control system. Several tomographic methods are available for soft X-ray or bolometric tomography, among which the Cormack and Neural networks methods stand out due to their inherent speed of up to 1000 reconstructions per second, with currently available technology. This paper discusses the application of these algorithms on fusion devices while comparing performance and reliability of the results. It has been found that although the Cormack based inversion proved to be faster, the neural networks reconstruction has fewer artifacts and is more accurate.
\end{abstract}

Keywords: tokamak, isttok, tomography, fourier-bessel, neural-network

PACS: $52.55 . \mathrm{Fa}, 42.30 . \mathrm{Wb}$

\section{INTRODUCTION}

A real-time plasma position control system is required to achieve long duration, alternating current discharges on the tokamak ISTTOK [1] $(R=0.46 \mathrm{~cm}, a=8.5 \mathrm{~cm})$. Such a system has been in place for some time using magnetic probes [2]. However, this system has been found to be limited during the current inversion of long duration, alternating current discharges. A tomography diagnostic has been installed to supply additional feedback to the control system. This paper presents two different tomographic algorithms which can be processed in real-time (one reconstruction in $0.1 \mathrm{~ms}$ ) using, for example, the Linux RTAI platform in a standard PC equiped with the acquisition boards.

The considered algorithms are the Fourier-Bessel $[3,4]$ and a neural-network (NN) method [5]. The Fourier-Bessel algorithm uses a fit of the available data to a set of basis functions: the Bessel functions are used in radial direction and the Fourier sine and cosine components representation for the poloidal direction. The NN is trained with phantom data to produce a given output for some predefined inputs. It will produce a good reconstruction if the presented problem is in some way an interpolation of the training set. 


\section{TOMOGRAPHY SYSTEM}

The ISTTOK tomography system consists of 30 photodiode channels, 10 in each of the 3 cameras (see fig. 1). For each 10, only the 8 innermost channels are actually connected to the acquisition system resulting in 24 used channels. Each camera has a circular pinhole of $1 \mathrm{~mm}$ and each photodiode has a size of $1.5 \times 1.5 \mathrm{~mm}$. The distance from the photodiode array to the pinhole is $1 \mathrm{~cm}$, allowing a full coverage of the plasma. No filter is used so each photodiode is essentially measuring visible light and some ultra-violet.

Each channel is amplified by a transductance amplifier with a gain of $1.8 \times 10^{6}$ and digitally acquired at a rate of 2 MSamples/s with 14 bits of resolution.

Since the power supplies that adjust the plasma position have a lag of the order of $100 \mu \mathrm{s}$ [6], not all the samples from the cameras are necessary to produce one tomogram in real-time, so only one sample every $128 \mu$ s is used, by filtering 256 samples.

\section{ALGORITHMS}

In tomography, the goal is to generate a cross-section view of a given medium from a set of integrated measurements.

In the fusion community, several tomography algorithms are used, the most common being the constrained regularization, also called pixel-based methods, Cormack based methods and Neural-Networks. The most common constrained regularization algorithms are the Maximum Entropy and the Minimum Fisher, but other simpler regularizations exist [7-9]. These algorithms are usually iterative, so they are not good candidates for a real-time application, although they do produce more reliable reconstructions than the other two algorithms. Because of that, this paper focuses on the Cormack method and $\mathrm{NN}$.

The Cormack method [10] essentially approximates the emissivity profile by a Fourier

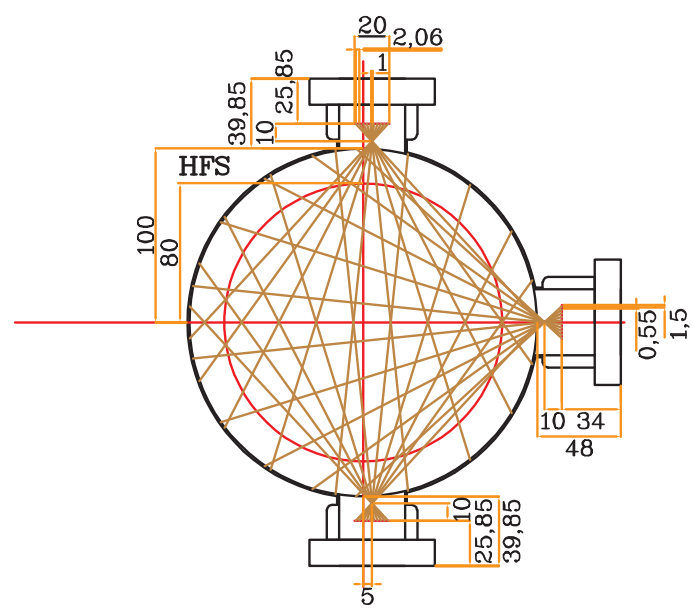

FIGURE 1. The tomography camera system of the tokamak ISTTOK: 3 cameras with 8 diodes each. Sight lines in the poloidal installation are drawn. 
expansion on the poloidal plane and an expansion in Zernicke polynomials in the radial direction. However, Zernicke polynomials have the inherent problem that they are non zero at the outer edge which can lead to completely unrealistic results. So a different type of functions must be used in the radial expansion and Wang [3, 4] found that the first order Bessel functions are appropriate.

\section{Fourier-Bessel basis functions}

The Radon transform in two dimensions can be written as

$$
f(p, \phi)=\int_{L(p, \phi)} g(r, \theta) d s
$$

where the integral path $L(p, \phi)$ is shown in fig. $2, g(r, \theta)$ is the plasma emissivity in poloidal coordinates and $f(p, \phi)$ is the measurement along the line $L$. Fourier expanding these two functions one can write them as

$$
\begin{gathered}
g(r, \theta)=\sum_{m=0}^{\infty} g_{m}^{c}(r) \cos (m \theta)+g_{m}^{s}(r) \sin (m \theta) \\
f(p, \phi)=\sum_{m=0}^{\infty} f_{m}^{c}(p) \cos (m \phi)+f_{m}^{s}(p) \sin (m \phi)
\end{gathered}
$$

Expanding further $g_{m}^{c, s}(r)$ one can obtain

$$
g_{m}^{c, s}(r)=\sum_{l=0}^{\infty} a_{m l}^{c, s} g_{m l}(r)
$$

Substituting eqs. (2), (3) and (4) into eq. (1) it can be shown that [10]

$$
f_{m}^{c, s}(p)=\sum_{l=0}^{\infty} a_{m l}^{c, s} f_{m l}(p)
$$

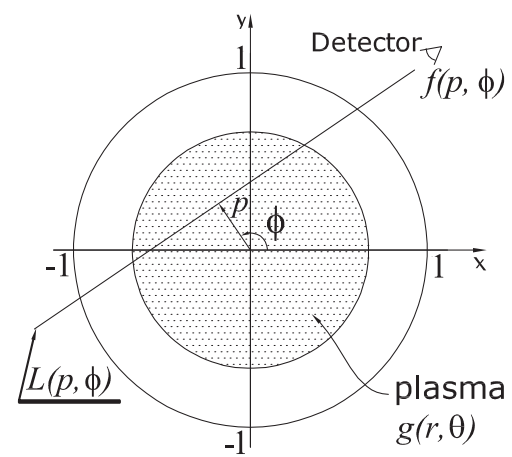

FIGURE 2. The measurement geometry of Eq.1. 
Now, if $g_{m l}(r)=\mathrm{J}_{m}\left(x_{m l} r\right)$, where $x_{m l}$ is the $(l+1)$ 'th zero of the first order bessel function $\mathbf{J}_{m}(x)$, according to [3], one can write

$$
\begin{aligned}
f_{m l}(p)=2 \sqrt{1-p^{2}} \mathrm{~J}_{m}^{\prime}\left(x_{m l}\right) & \\
& \times \sum_{n=0, m \neq n}^{\infty} \delta_{n} \mathrm{~J}_{n}\left(x_{m l}\right) \sin \left(n \frac{\pi}{2}-x_{m l} p\right)\left(\frac{U_{m+n-1}(p)}{m+n}+\frac{U_{m-n-1}(p)}{m-n}\right)
\end{aligned}
$$

where $\delta_{n}=\frac{1}{2}$ for $n=0$ and $\delta_{n}=1$ otherwise. $U_{m}(p)$ is the Chebyshev polynomial of the second kind, $U_{m}(p)=\frac{\sin \left[(m+1) \cos ^{-1} p\right]}{\sqrt{1-p^{2}}}$. It is impractical to follow these summations to infinity, so they must be truncated. This truncation is done according to the following criteria:

- The $l$ index is associated with the radial component of the expansion. The resolution of this component is determined by the number of photodiodes in each camera, according to the Nyquist theorem; in the present case of 8 photodiodes per camera, $N_{l}=4$, or $l=0,1,2$ and 3 .

- The $m$ index is associated with the angular component of the expansion and its resolution is determined by the number of cameras. For 3 equidistant cameras, one can have up to $m=2$, but since ISTTOK's cameras are not equidistant $N_{m}=2$, or $m=0$ and 1 .

If all $a_{m l}^{c, s}$, s are known, then one can use eqs. (4) and (2) to generate the tomographic reconstruction. Each photodiode's position is given by $\left(p_{i}, \phi_{i}\right)$, so one can write $f_{i}=$ $f\left(p_{i}, \phi_{i}\right)$. By writing $X_{i, m l}^{c}=f_{m l}\left(p_{I}\right) \cos \left(m \phi_{i}\right)$ and $X_{i, m l}^{s}=f_{m l}\left(p_{i}\right) \sin \left(m \phi_{i}\right)$ one can use the matrix notation $\vec{f}=\mathbf{X} \cdot \vec{a}$ or:

$$
\left[\begin{array}{c}
f_{1} \\
f_{2} \\
\vdots \\
f_{N}
\end{array}\right]=\left[\begin{array}{ccc}
X_{1,00}^{c} & \cdots & X_{1,13}^{s} \\
X_{2,00}^{c} & \cdots & X_{2,13}^{s} \\
\vdots & & \vdots \\
X_{N, 00}^{c} & \cdots & X_{N, 13}^{s}
\end{array}\right] \cdot\left[\begin{array}{c}
a_{00}^{c} \\
a_{01}^{c} \\
\vdots \\
a_{13}^{c} \\
a_{10}^{s} \\
\vdots \\
a_{13}^{s}
\end{array}\right]
$$

Since this is a fitting problem with more equations than unknowns, $\mathbf{X}$ is not a square matrix so a simple matrix inversion is not possible. A least squares fit can be performed using the Singular Value Decomposition (SVD) of the matrix $\mathbf{X}=\mathbf{U W V}^{\mathrm{T}}$ [11]:

$$
\vec{a}=\sum_{i=0}^{M} \frac{\vec{U}_{i} \cdot \vec{f}}{W_{i}} \cdot \vec{V}_{i}
$$

where $\vec{U}_{i}\left(\vec{V}_{i}\right)$ is the $i$ 'th column of the matrix $\mathbf{U}(\mathbf{V}), W_{i}$ is the $i$ 'th diagonal value of the matrix $\mathbf{W}$ and $M$ is the number of columns of $\mathbf{X}$. Since $\mathbf{X}=\mathbf{X}(p, \phi)$, it can be precalculated as well as it's SVD. Thus, for each tomogram, only eq. (8) needs to 


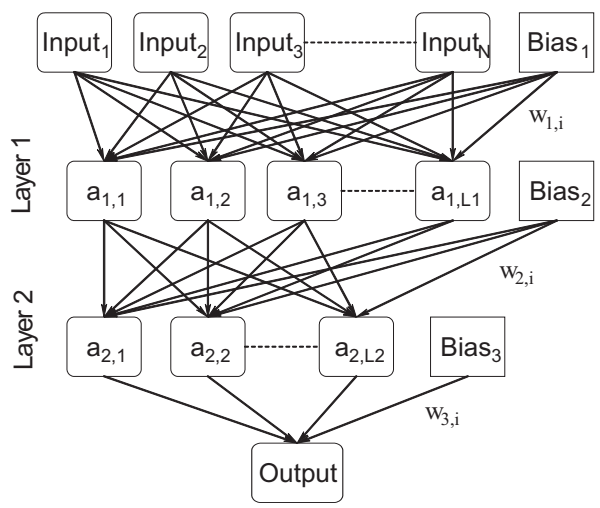

FIGURE 3. A multilayer perceptron Neural Network, with 2 hidden layers. Each neuron is a function of all neurons on the previous layer and the bias. The bias neuron is independent of previous layers.

be calculated and the reconstruction can then be built on any $(x, y)$ grid. In this case, a $33 \times 33$ grid was used. It has more pixels than the system can resolve, but not as many as to take too long to build a reconstruction.

\section{Neural Networks}

A Neural Network (NN) consists of simple processing units (neurons) connected amongst each other in a particular way. Typically, a NN is arranged in layers of neurons, where any one neuron can have a connection to all other neurons on the previous layer (see fig. 3). Each neuron has one activation function which can be any function of neurons from the previous layer(s). The activation function $z$ used in this work is $z=\tanh \left(\sum_{i} w_{i} a_{i}\right)$ where $a_{i}$ is the output from the neuron $i$ of the previous layer and $w_{i}$ is the weight that neuron $i$ has on the present neuron.

There are many types of NNs. In this work, a multi-layer perceptron was used. A NN must be trained in order to determine the weights. This training is done with an artificial input and output data set. For tomography, one generates a set of phantoms of expected spacial radiation distributions of the plasma and, using virtual sensors on this phantom, generates the corresponding sensor data. For this work, the phantoms generated were rings of different widths, radii and positions, because it makes sense that the $H_{\alpha}$ radiation is coming from the outer part of the plasma.

The perceptron networks have one extra neuron in each layer which isn't related to the previous layers; this is the bias neuron and is usually set to 1 . The training and layer optimization of the network was performed using bayesian methods [5] and produced a two hidden layer network where the first hidden layer has 9 neurons and the second hidden layer also has 9 neurons. The inputs for the network are the data channels from the photodiodes and the $(x, y)$ position of the pixel being reconstructed and the output is the value of this pixel. So for each reconstruction, the NN must run for as many times as there are pixels in the reconstruction grid $(33 \times 33=1089$ runs $)$. 


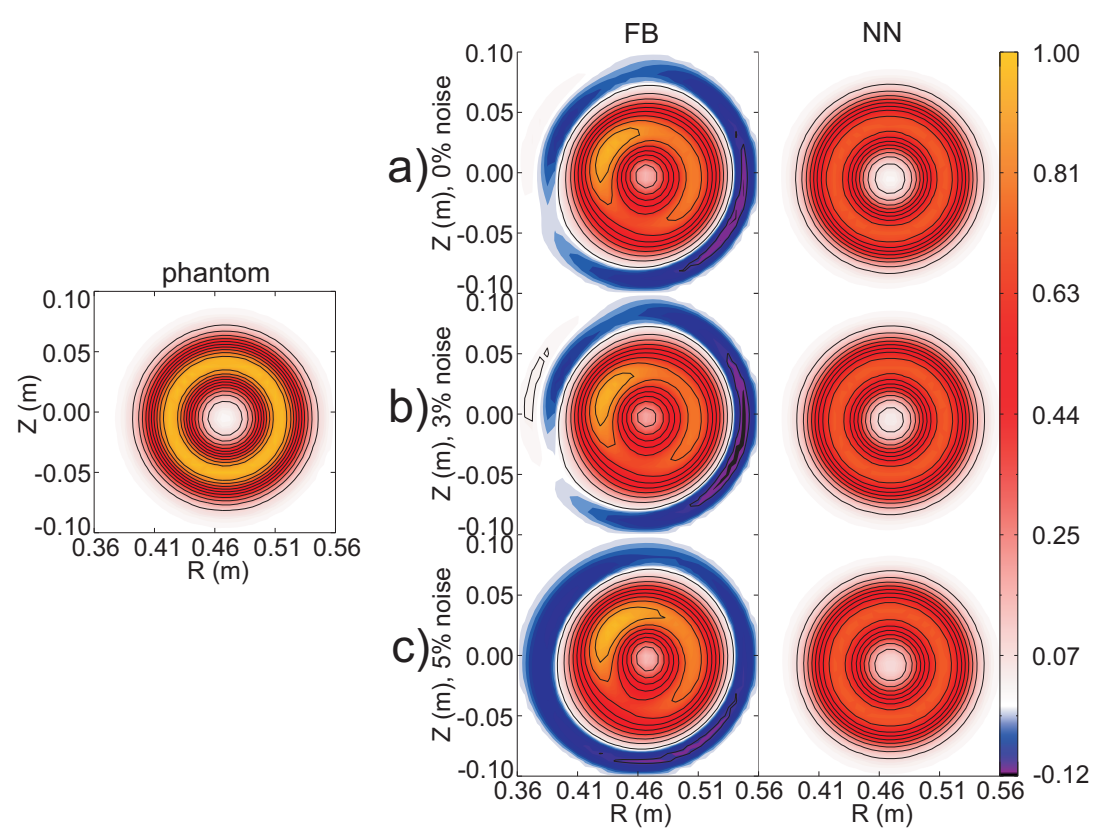

FIGURE 4. Comparison of the reconstructions using Fourier-Bessel (FB) and Neural Networks(NN) algorithms contemplating different ranges of measurement noise - a) has no noise, $b$ ) has $3 \%$ noise and c) has $5 \%$ noise. Clearly, the NN reconstructions are superior to the FB in any of the cases presented. The phantom used (right) is a ring slightly shifted from the center by $8.6 \mathrm{~mm}$ in $R$ and $-5 \mathrm{~mm}$ in $Z$.

\begin{abstract}
ANALYSIS
The algorithm speed tests were run on a standard PC running the Real Time Application Interface (RTAI [12]) for Linux, with an Intel®Pentium®4 Processor at a clock speed of $2.80 \mathrm{GHz}$. RTAI is basically an interrupt dispatcher, trapping the peripherals' interrupts and, if necessary, re-routing them to Linux. One can have a task running on a given processor without being interrupted until this task is finished so that it will always take the same time to run.

ISTTOK's tomography systems's lines of sight geometry was used to generate the virtual channel data for processing by the algorithms.

For a reconstruction on a $33 \times 33$ grid, the Fourier-Bessel Algorithm takes about $40 \mu \mathrm{s}$ and the NN takes $356 \mu \mathrm{s}$, using only Long numbers in KERNEL space.

Figure 4(a) shows the test emissivity (phantom) and the reconstructions of it using both algorithms discussed. The phantom used is a ring slightly off-centered.

Table 1 shows the error of the reconstructions for the same noise levels as in figure 4. Since the noise is added randomly, 10 different runs were performed to have some statistics. The error presented is the mean of these 10 runs. For each run, it was calculated using $\varepsilon=\frac{\sum_{x, y}\left(g_{p h}(x, y)-\hat{g}(x, y)\right)^{2}}{\sum_{x, y}\left(g_{p h}(x, y)\right)^{2}}$ where $g_{p h}$ is the phantom and $\hat{g}$ is the reconstruction.

The standard deviation of the error was also calculated and it was found that the FB algorithm presents less sensitivity to the noise.

Figure 4(b) shows the reconstructions when the signals have 3\% random gaussian
\end{abstract}


TABLE 1. Errors of the reconstructions for both FourierBessel and Neural-Netowrks algorithms, with different noise levels added to the channel data. The standard deviation of the error is also presented. $d$ is the distance in centimeters of the center of mass of the phantom to the center of mass of the reconstruction presented in figure 4.

\begin{tabular}{|c||ccc|ccc|}
\hline \multirow{2}{*}{ noise } & \multicolumn{3}{|c|}{$\mathrm{FB}$} & \multicolumn{3}{c|}{$\mathrm{NN}$} \\
& $\varepsilon$ & $\sigma$ & $d(\mathrm{~cm})$ & $\varepsilon$ & $\sigma$ & $d(\mathrm{~cm})$ \\
\hline $0 \%$ & 0.085 & - & 0.422 & 0.036 & - & 0.008 \\
$3 \%$ & 0.086 & 0.002 & 0.445 & 0.046 & 0.017 & 0.082 \\
$5 \%$ & 0.087 & 0.003 & 0.390 & 0.047 & 0.017 & 0.255 \\
\hline
\end{tabular}

noise added and figure 4(c) shows the reconstructions when the signals have 5\% random gaussian noise added.

One algorithm to determine the plasma position can be the center of mass of the reconstructed emissivity. The column labelled $d$ in table 1 was built using this algorithm and calculating the distance between the phantom's center of mass and the reconstructions'. Again, it is apparent that the NN algorithm produces a better reconstruction than the FB algorithm, with a deviation of less than $1 \mathrm{~mm}$ versus $4.5 \mathrm{~mm}$, for $3 \%$ random noise.

\section{DISCUSSION}

The Fourier-Bessel algorithm was 9 times faster than the Neural-Network, for the same setup. The FB algorithm has shown to be more robust in the presence of noisy data as shown by the lower standard deviation of the tests. However, the NN produces better reconstructions, almost perfectly reconstructing the proposed phantom. Considering the need for a reconstruction in each $128 \mu \mathrm{s}$, only the FB is suitable and it still allows for some time to post-process the reconstruction and determine the plasma position or other parameters that may be obtainable. On the other hand, a NN dedicated to determine the position, instead of the whole reconstruction, would be much faster and there would be no need for a post-processing step.

Determining the center of mass of the reconstruction, it was found that the NN reconstruction presents a better agreement with the phantom for up to $5 \%$ noise in the data.

One further remark is that the NN can only reconstruct what it's been trained to, while the FB reconstructs only what is allowed by the maximum order of the Bessel functions and the Fourier modes - the maximum $m$ and $l$ discussed in section. As a result, the NN's reconstruction can fail if the channel data is too different from the training set, although this may be detected [5], while the FB's has always some artifacts which must be filtered by a post-processing algorithm.

Taking into consideration the present hardware capabilities at ISTTOK, was shown that the FB algorithm is the most suitable method for plasma position control application due to the reconstruction time constraints.

The application of these methods to the experimental data is ongoing. 


\section{ACKNOWLEDGMENTS}

This work has been carried out within the framework of the Contract of Association between the European Atomic Energy Community and "Instituto Superior Técnico". Financial support was also received from "Fundação para a Ciência e Tecnologia" in the frame of the Contract of Associated Laboratory.

\section{REFERENCES}

1. C. Varandas, et al., Fusion Technology 29, 105 (1996).

2. D. Valcárcel, et al., Real-time plasma position control on the isttok tokamak (2007), proposed for the Fusion Engineering and Design journal.

3. L. Wang, and R. S. Granetz, Review of Scientific Instruments 62, 1115-1116 (1991).

4. L. Wang, and R. S. Granetz, Review of Scientific Instruments 62, 842-843 (1991).

5. U. v. Toussaint, et al., Neural Networks 19, 1550-1557 (2006).

6. I. Carvalho, et al., Fast controlled current sources for plasma stabilization (2007), proposed for the IEEE Transactions on Industrial Electronics journal.

7. M. Anton, et al., Plasma Physics and Controlled Fusion 38, 1849-1878 (1996).

8. $\quad$ K. Ertl, et al., Nuclear Fusion 36, 1477-1488 (1996).

9. L. C. Ingesson, and JET EFDA contributors, "Tomography methods in plasma diagnostics," in Tomography Symposium, Meeting of the Japanese Physical Society, Japanese Physical Society, 2003.

10. A. M. Cormack, Journal of Applied Physics 35, 2908 (1964).

11. W. H. Press, S. A. Teukolsky, W. T. Vetterling, and B. P. Flannery, Numerical Recipes in C, The Art of Scientific Computing, Ch. 15.4, Cambridge University Press, 1992, second edn.

12. RTAI Team, Real time application interface (2007), URL https : / / www . rtai . org/. 